\title{
EN BUSCA DE LA AFIRMACIÓN: SEGURIDAD Y BRICS EN LA GOBERNANZA INTERNACIONAL
}

\author{
Marcelo de Almeida Medeiros \\ Mikelli Marzzini L. A. Ribeiro \\ Mariana P. O. DE LyRA
}

\section{INTRODUCCIÓN}

Las relaciones internacionales se desarrollan, en gran parte, por reglas que tienen su origen en la praxis de los actores internacionales. Tales reglas han surgido y son administradas por un grupo restringido de potencias que busca mantener un determinado orden internacional. A partir del final de la Guerra Fría, las potencias occidentales se erigieron como las gestoras del sistema mundial. Sin embargo, el ascenso de las llamadas potencias emergentes ${ }^{1}$ ha mostrado la posibilidad de un reacomodo en la distribución del poder. Este ascenso se caracteriza por buscar su participación en asuntos menos sensibles (low politics) y en aquellos que son más sensibles (high politics).

Brasil, Rusia, India, China y África del Sur (BRICS), como potencias emergentes, pueden considerarse entre los estados más prominentes. Según ascienden éstos en el sistema internacional, procuran ejercer más influencia en la gobernanza global, incluso si están agrupados en torno de los BRICs. ${ }^{2}$ Situación evidenciada en lo que atañe a los asuntos económicos. Es más: en los recientes

${ }^{1} \mathrm{El}$ concepto de potencias adoptado en este estudio se detallará más adelante.

${ }^{2}$ A. Hurrell ("Hegemonia, liberalismo e ordem global: qual é o espaço para potências emergentes?”, en A. Hurrell et al., Os BRICS e a ordem global, Rio de Janeiro, Editora FGV, 2009, p. 11) afirma que "todos esos países comparten la idea en su derecho a un papel más influyente en asuntos mundiales". 
encuentros del grupo se observa que, ampliando la agenda, se han alcanzado los asuntos de high politics -en especial, la seguridad.

La bibliografía especializada reciente sobre los BRICS ha procurado demostrar la importancia de los asuntos de seguridad para el grupo. Hay tanto estudios que disienten de la relación de los BRICS con temas específicos, tales como cuestiones nucleares ${ }^{3}$ y el papel del Consejo de Seguridad, ${ }^{4}$ como análisis que investigan, de manera general, la llamada security policies de sus miembros. ${ }^{5}$

El aumento de la producción académica sobre temas de seguridad se asocia a la ampliación del propósito de la agenda del grupo. Es importante entender, entonces, la medida en que tales temas están incluidos en esa nueva agenda extendida. Este artículo investiga, por ello, en qué forma el BRICs se ha enarbolado para buscar una gestión más amplia de las reglas internacionales a partir de sus posicionamientos conjuntos, en particular los relacionados con la seguridad internacional. En los últimos años, se ha intentado dosificar el aumento de los asuntos sobre seguridad en las declaraciones del BRICS, así como reflexionar sobre su contenido.

Se defiende la hipótesis de que el BRIcs busca su afirmación y se sabe capaz de influir en los asuntos más sensibles de la gobernanza internacional, partiendo del aumento en la agenda del grupo en asuntos sobre seguridad. Se utilizan, para demostrarlo, los supuestos establecidos por la Escuela Inglesa, en especial la idea de que son los actores centrales de la sociedad internacional (superpotencias y grandes potencias) los únicos que pueden administrar cuestiones ligadas a las high politics, en especial la seguridad.

${ }^{3}$ Véase M. Herz y V. Coutinho Lage, "Brics e a Questão Nuclear: contestações e reafirmações diante dos mecanismos de governança global”, Carta Internacional, vol. 6, núm. 2, 2011, pp. 31-54.

${ }^{4}$ Véase O. Stuenkel, "The BRICs on the un Security Council: The Case of R2P”, The Brics and the Future of Global Order, Lanhuam, Lexington Books, 2015, pp. 125-145.

${ }^{5}$ Véase M. Troitskiy, "BRIcs Approaches to Security Multilateralism”, ASPJ Africa Eं Francophonie, $2^{\text {nd }}$ Quarter, 2015, pp. 76-88. 
Referirse a "evidencias significativas" implica decir que la confirmación empírica del levantamiento aquí propuesto no es exhaustiva, pero sirve de base para estudios posteriores que busquen, por fuentes diversas, reforzar tal afirmación.

Las fuentes centrales son, por tanto, las declaraciones de cúpula del grupo, en un horizonte temporal que va de 2009, año en que se llevó a cabo la primera reunión (aún no participaba África del Sur), hasta la cúpula llevada a cabo en 2015. Se ha utilizado el método cualitativo de análisis de documentos asociado a la cuantificación simple de palabras-clave.

El estudio se dividió en tres secciones. La primera discute las premisas teóricas de la Escuela Inglesa en lo relacionado con la gobernanza, resaltando el papel de las potencias en la gestión del orden internacional. Se ha profundizado luego en el caso particular del surgimiento de los BRICs y su función en la gobernanza internacional. Y, en la última sección, se investiga el aumento de los temas sobre seguridad en la agenda del BRICS. Se desarrolló, así, un análisis que busque tal afirmación y que el grupo sea visto como polo relevante en el concierto político contemporáneo, con capacidad de administrar las reglas más sensibles de la esfera internacional.

\section{LA ESCUELA INGLESA Y LA GOBERNANZA INTERNACIONAL}

La Escuela Inglesa (EI), uno de los campos teóricos más fértiles en las Relaciones Internacionales (RI), procura establecer un diálogo más allá de los límites de las tradiciones realista e idealista de la disciplina. Se considera una vía media ${ }^{6}$ que incorpora elementos de poder e institucionales, que se basa en la premisa de una sociedad interestatal, en la cual hay ciertas instituciones, normas y reglas comunes. ${ }^{7}$ Las normas son expresiones que "dicen lo que son los estados y otros grupos, a qué lugar pertenecen y qué tipo

${ }^{6}$ Véase B. Buzan, “An Underexploited Resource in IR”, Review of International Studies, vol. 27, núm. 3, 2001, pp. 471-488.

${ }^{7}$ Véase H. Bull, A Sociedade Anárquica: um estudo da ordem na política mundial, trad. de Sérgio Bath, Brasilia, Editora Universidade de Brasilia, 2002. 
de papel ejercen". ${ }^{8}$ Muestran un grado de organización que no podría identificarse normalmente en un sistema anárquico. ${ }^{9}$

La estructura normativa proporciona un orden para la arena internacional, un orden interestatal. Según Bull, ${ }^{10}$ se caracteriza por ser un arreglo específico, cuya función es dirigir las acciones de los actores internacionales, de modo tal que los objetivos primordiales de la sociedad internacional puedan alcanzarse con mayor facilidad. Un aspecto relevante presente en la idea del orden internacional es el papel que el autor clasifica como grandes potencias, las cuales se derivan "de un simple hecho, la desigualdad de poder entre los estados que participan del sistema internacional". ${ }^{11}$

Bull también señala que para clasificar una gran potencia deben observarse tres características implícitas. La primera se relaciona con la existencia de dos o más potencias de status semejante, "como si existiera un club cerrado con una regla escrita para admitir a los socios". ${ }^{12}$ La segunda se refiere a la idea de que los estados considerados como grandes potencias ocuparían el primer plano en lo que toca al poder militar. La última corresponde a la noción de que éstas tienen ciertos derechos y obligaciones especiales -derivados del reconocimiento de su propio pueblo y de su liderazgo frente a otros estados.

Es evidente que la sociedad internacional en la época que se publicó A sociedade anarquica, 1977, era otra. ${ }^{13}$ Comprender esa sociedad es necesariamente entender que había un club de estados más fuerte que regulaba el orden internacional.

En ese sentido, Buzan y Waever ${ }^{14}$ argumentan que los estados que tienen cierto relieve en el sistema internacional, sobre todo en

${ }^{8}$ A. Hurrell, On Global Order: Power, Values, and the Constitution of International Society, Nueva York, Oxford University Press, 2007, p. 38.

${ }^{9}$ Véase A. Linklater y H. Suganami, The English School of International Relations: A Contemporary Reassessment, Cambridge, University Press, 2006.

${ }^{10}$ Véase H. Bull, op. cit.

11 Ibidem, p. 235.

12 Ibid., p. 329.

${ }^{13}$ En la clasificación de Bull (op. cit.) existían tres grandes potencias en ese periodo: Estados Unidos, Unión Soviética y China.

${ }^{14}$ Regions and Powers. The Structure of International Security, Cambridge, University Press, 2003. 
lo relacionado con la seguridad, pueden clasificarse en tres niveles: 1) superpotencia; 2) gran potencia; 3) potencia regional. La superpotencia es la que tiene intereses globales diversos, además de reunir una gran capacidad de defensa tanto en términos de medios disponibles para asegurar sus intereses, como en alcance geográfico. Estados Unidos y la extinta Unión Soviética son ejemplos de superpotencias. Las grandes potencias son los Estados con amplio alcance, pero sin llegar a lo global, es decir la gran potencia tiene intereses diversos, pero no cuenta con los medios para defenderlos globalmente. Y la potencia regional es fundamental para la dinámica y el equilibrio de una región determinada mientras no ejerza un papel sistémico.

La gobernanza internacional ignora la gerencia de las principales potencias en el ámbito de los estados. Para Hurrell, la gobernanza actual se centra en una estructura oligárquica "construida en torno de un grupo relativamente pequeño de grandes potencias occidentales". ${ }^{15}$ Dado que en este momento la sociedad internacional atraviesa por una transición de poder, aparece un proceso reciente que busca el reacomodo del orden mediante la inserción de potencias emergentes ${ }^{16}$ en la gobernanza internacional.

En la bibliografía especializada sobre política internacional, se tiene una percepción de las potencias emergentes como de estados de crecimiento relativo en la distribución de la riqueza global, cuyas ganancias se convierten en poder político. La conversión puede evaluarse luego de ese aumento de riqueza, porque se adquiere capacidad militar, y porque además se constituye un liderazgo regional o se establece un orden normativo más favorable

15 "Narratives of emergence: Rising powers and the end of the Third World?", Brazilian Journal of Political Economy, vol. 33, núm. 2, 2013, p. 251. En este artículo se supone que tal grupo está compuesto, en especial, por la única superpotencia (Estados Unidos) y las grandes potencias europeas (Francia, Gran Bretaña y Alemania).

${ }^{16}$ Véase A. Hurrell, "Power Transitions, Emerging Powers, and the Shifting Terrain of the Middle Ground", en Cornelia Navari (ed.), Ethical Reasoning in International Affairs: Arguments from the Middle Ground, Londres, Palgrave Macmillan, 2013, pp. 222-245. 
durante el proceso mismo. ${ }^{17} \mathrm{El}$ actual grupo de países emergentes se compone en especial por potencias regionales que pertenecen a Occidente, como Brasil, África del Sur, Indonesia y Turquía, por lo que en esa circunstancia entran también casos particulares como Rusia y China. ${ }^{18}$

Las potencias occidentales -estados centrales en la gobernanza en el orden actual- aceptan a las potencias emergentes mientras esperan el ajuste de la sociedad internacional. ${ }^{19}$ Tal aprobación es parte, en cierta medida, de la actuación política de las nuevas potencias, para así obtener mayor reconocimiento. Hurrell revela este fenómeno al referirse a Brasil, Rusia, India y China:

El reconocimiento que persiguen esos cuatro países es parte fundamental de la dinámica política en un ambiente mundial de jerarquías. Los desafíos a la legitimidad del orden internacional rara vez dan resultados si las protestas provienen de economías débiles; con mayor frecuencia han llegado de los Estados o pueblos con capacidad y organización política suficientes para exigir la revisión del orden establecido y de sus normas dominantes. ${ }^{20}$

${ }^{17}$ Para el concepto de potencias emergentes, véase P. C. Dutra Fonseca, L. de Oliveira Paes y A. Moreira Cunha, "The concept of emerging power in international politics and economy”, Revista de Economia Política, vol. 36, núm. 1, 2016, pp. 46-69.

${ }^{18} \mathrm{El}$ grupo es particularmente importante para las potencias regionales: Brasil, India y África del Sur. En el caso de Rusia y China, el peso del grupo es relativamente menor. Esos dos estados son considerados grandes potencias por buena parte de estudiosos, como Buzan y Waever (op. cit.). Rusia, una gran potencia con sus capacidades materiales e influencia política heredada de la extinta Unión Soviética; China, a su vez, el estado con mayor poder relativo entre ellos y un proceso de consolidación internacional. En el orden vigente, sin embargo, dominado por una "oligarquía de grandes potencias occidentales" (Hurrell, "Power Transitions, Emerging Powers...”), esas dos potencias encontraron en el grupo una concertación para alcanzar mayor legitimidad de actuación en la gobernanza global.

${ }^{19}$ Como ejemplos de esa aceptación se tiene la creciente importancia del G-20, en especial en la crisis posterior de 2008; la inclusión de Brasil y África del Sur como partícipes estratégicos de la Unión Europea (UE), así como el fortalecimiento de China, Rusia e India, y la uE. Para mayor detalle, véase http:/ /strategicpartenerships.eu

20 "Hegemonia, liberalismo e ordem global...", p. 11. 
El papel de esos actores recae en especial en la discusión de ciertos temas específicos (Brasil y la India en la cuestión ambiental y comercial, por ejemplo). Sin embargo, según aumenta la legitimidad, se tiende a intervenir en los asuntos de high politics ligados a la seguridad internacional. ${ }^{21}$ Es lo que sucede, en apariencia, cuando se actúa de forma concertada, como ocurre en el BRIcs.

En ese sentido, los estudios de gobernanza que se fundamentan en la EI incluyen reflexiones importantes, toda vez que puede inferirse, a partir de la visión de Bull en su obra citada, que son las grandes potencias las únicas que tendrían la legitimidad internacional para discutir sobre normas de seguridad, por tratarse de un tema central para el orden vigente. ${ }^{22}$ Con fundamento en las premisas de la EI, es posible entender que el BRICs, además de buscar su legitimidad, se siente con la capacidad para entrar en el concierto de la regulación de las normas más sensibles de la sociedad internacional.

Se intenta analizar, en este artículo, la inserción de los temas de seguridad en la agenda del BRICs, visto que tal asunto se concibe como el más sensible en el orden internacional. Pero antes, es conveniente presentar un breve análisis de la actuación del grupo en lo tocante a la gobernación internacional, de forma general, lo que se presenta de manera sucinta en la siguiente sección.

\section{EL SURGIMIENTO DE LOS BRICS Y LA GOBERNANZA INTERNACIONAL}

El aumento del BRICs como nuevos agentes de la gobernanza internacional es parte de uno de los grandes debates en las RI, porque

${ }^{21}$ Véase Hurrell, op. cit.

${ }^{22}$ Bull (op. cit.) presenta una visión plural del orden internacional, predominantemente centrada en el estado. Los pluralistas ven en los derechos de los estados, como la soberanía y la no intervención, los aspectos centrales de ese orden. Para el autor, hay tres objetivos principales para el orden internacional: $a$ ) preservar la sociedad de estados, $b$ ) mantener la existencia individual de los estados y $c$ ) regular la violencia (o uso de la fuerza). Desde esa perspectiva, dos valores estarían situados en el centro del orden internacional: sobrevivencia y coexistencia. Partiendo de su importancia dentro de esa lógica, se infiere que normas y reglas de seguridad deben ser administradas por las grandes potencias. Para más información sobre pluralismo/solidaridad en el orden internacional vigente, véase Hurrell, op. cit. 
incluye los cambios recientes en el sistema internacional. ${ }^{23}$ Diversos analistas defienden la teoría de que se vive una transformación: se abandona un sistema centrado en Estados Unidos para dirigirse a un orden multipolar, ${ }^{24} \mathrm{o}$ un mundo posthegemónico ${ }^{25}$, en el que la superpotencia norteamericana cedió espacio a otros polos de poder.

Si se analiza esa estructura cambiante, las transformaciones del sistema internacional se configuran como la confluencia de tres tendencias: 1) el poder económico se ha distribuido mucho más rápidamente que el poder político en los últimos 30 años; 2) los procesos de regionalización se han consolidado como vía de desarrollo de los estados y 3) el surgimiento de algunas potencias regionales. La interacción de estas tres vertientes ha causado cambios en la estructura política y económica global, en la que las potencias regionales adquirieron más autonomía para intervenir en la agenda internacional. El BRICs sería uno de los productos más evidentes de este proceso contemporáneo.

Originado a partir de dos artículos desarrollados por el banco Goldman Sachs (Building Better Global Economic BRICs y Dreaming with BRICS: The Path to 2050), ${ }^{26}$ al grupo formado por Brasil, Rusia, India, China y, más tarde, África del Sur, se dio trato de conglomerado de países extremamente promisorios en un periodo de 50 años, cuyas economías sumadas podrían superar al Norte, rico e industrializado, en $2050 .{ }^{27}$

${ }^{23}$ Para el papel que desempeñan los BRICs en la reestructura de la gobernanza internacional, véase Z. Hour, "The BRICs and Global Governance Reform: Can the BRICS provide leadership?”, Development, vol. 3, núm. 56, 2013, pp. 356-362.

24 Stephen M. Walt, "The end of the American era”, The National Interest, núm. 116, 2011, pp. 6-16.

${ }^{25}$ Véase F. Zakaria, O mundo pós-americano, trad. de Pedro Maia, São Paulo, Companhia das Letras, 2008.

${ }^{26}$ G. Fonseca Jr. (“Brics: notas e Questões”, en J. V. de Sá Pimentel, org., $O$ Brasil, os BRICS e a Agenda Internacional, Brasilia, Funag, 2012 O Brasil, os BRICS e a Agenda Internacional, Brasilia, Funag, 2012, pp. 13-30) argumenta que "es natural que, como casa de bolsa, el centro de la reflexión de Goldman Sachs fuera el interés de sus clientes. Hacer el acrónimo era una solución rápida y eficaz para recordar dónde estarían, en el medio y largo plazos, las buenas oportunidades”.

${ }^{27}$ J. O’Neill, "Building Better Global Economic brics", GS Global Economics, Paper 66, noviembre de 2001, en http://www.goldmansachs.com/our-thinking/ archive/archive-pdfs/build-better-brics.pdf 
La formalización del BRICs se dio en 2009, en Ecaterimburgo, Rusia, donde se llevó a cabo la I cúpula del BRIC. El propósito del encuentro, además de su formalización, fue iniciar discusiones para lograr un orden mundial más democrático y multipolar. Fue hasta 2011 que el grupo incluyó a África del Sur y, con ello, se consolidó el acrónimo BRICs. ${ }^{28}$

Los estados que integran el grupo representan el $43.03 \%$ de la población mundial; el 18\% del Producto Interno Bruto (РIB) nominal mundial; el 25\% del Рів per capita; el $25.91 \%$ del espacio terrestre del planeta; y fueron responsables del $46.3 \%$ del crecimiento económico global entre los años 2000 y 2008. ${ }^{29}$ Además, cuentan con gastos militares significativos; en 2015, el total de su valor ascendía a 360509 millones de dólares. ${ }^{30}$

En las dos últimas décadas, han presentado un rápido crecimiento económico y cambios acelerados. China se consolidó, en este escenario, como la segunda economía mundial más importante. Rusia superó la crisis de 1998. Brasil dio grandes pasos en su desarrollo económico. La India emprendió cambios significativos en su economía y África del Sur figura como una de las grandes fuerzas de su continente.

Como lo reseña Gelson Fonseca Jr., en su estudio arriba mencionado, la formación de este grupo va más allá de semejanzas relacionadas con posiciones de poder. Lo que los aproxima, además de las oportunidades de inversión, son las posibilidades del ejercicio de poder. Así es que el grupo se creó no sólo para establecer oportunidades mutuas, sino como un aparente intento de reconocimiento con la intención de participar más activamente

${ }^{28}$ Véase M. E. F. Reis, “BRICs: surgimento e evolução”, en J. V. de Sá Pimentel (org.), op. cit., pp. 31-47.

${ }^{29}$ Ibid.

${ }^{30}$ La suma asciende a casi el $83 \%$ de la inversión de Estados Unidos en el mismo año (433 220 mdd). Vale mencionar que África del Sur tiene una cifra porcentual muy inferior a la de los demás (datos individuales de 2015 en millones de dólares: Brasil, 31 954; Rusia, 91 081; India, 51 116; China, 214 485; África del Sur, 3460$)$. Stockholm International Peace Research Institute ha hecho esta investigación, que se encuentra disponible en https//www.sipri.org/sites/default/files /Milex-constant-USD.pdf 
de la administración del orden internacional. Eso se reveló, en cierta medida, en sus posicionamientos conjuntos, a partir de las reuniones periódicas en las que se establecieron temas y prioridades de la agenda del grupo.

Desde 2009, año de la primera cúpula, se han llevado a cabo siete reuniones de jefes de Estado. En estas reuniones, se ha buscado mantener la rotación de los grupos locales y adoptar un tema rector. Además de organizar y efectuar las cúpulas, el grupo también mantiene encuentros regulares de académicos ${ }^{31}$ y ministros de Estado. ${ }^{32}$ El BRICs promueve desde 2009 el encuentro de Altos representantes sobre seguridad o asesores sobre seguridad nacional. ${ }^{33}$

El grupo, en sus inicios, se concentró en cuestiones económicas encaminadas a buscar cambios en las estructuras macroeconómicas mundiales, una mayor participación en organismos financieros internacionales (Fondo Monetario Internacional y Banco Mundial) y en los grandes foros e instituciones (G-20, Organización Mundial del Comercio). ${ }^{34}$ Como ejemplo, se observa que los asuntos económicos se tomaron en cuenta en las agendas de todas las reuniones de cúpula en las que había temas definidos (a partir de la cuarta): "estabilidad global" y "prosperidad" (2012); "desarrollo, integración e industrialización" (2013); "crecimiento" y sustentabilidad en la sexta, y "desarrollo global” (2015) (véase cuadro 1). Lo que resulta aún más sintomático si se constata que se creó un banco de desarrollo a partir de la sexta reunión de cúpula.

${ }^{31}$ Los foros académicos de los BRICS consisten en reuniones anuales llevadas a cabo antes de las cúpulas de los BRICs, con participación de profesores y académicos de los países miembros. Los objetivos de esos foros son: “1) profundizar la colaboración en términos de estudios de interés mutuo; 2) establecer redes entre comunidades académicas de los cinco países y 3) ofrecer resultados a los líderes de los BRICS de estudios empíricos" (IPEA, O Fórum-2014, en http://www.ipea.gov.br/ forumbrics/pt-BR/o-forum.html).

${ }^{32}$ Es práctica de los BRICs tener encuentros de ministros de diversas áreas, como relaciones exteriores, ciencia y tecnología y de hacienda. La agenda y relatorías de esos encuentros pueden consultarse en los websites oficiales de cada cúpula.

${ }^{33}$ La agenda y relatoría de esas reuniones no se divulgaron, por desgracia.

${ }^{34}$ Reis, art. cit. 


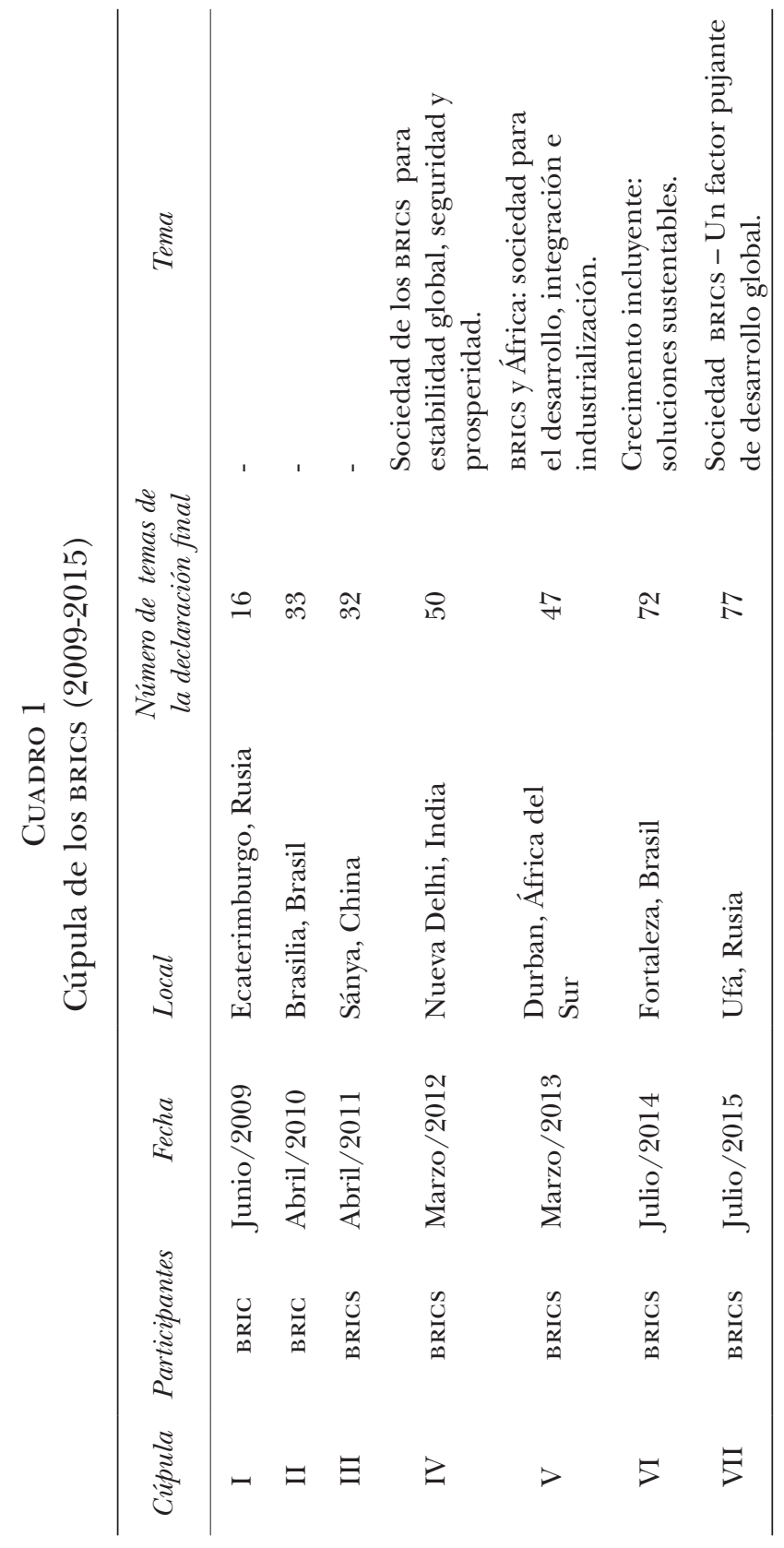


Los aspectos económicos y financieros continúan dictando buena parte de la agenda en las declaraciones de cúpula. La presencia de este tema refleja un aumento cuantitativo, así como un detalle más minucioso de tales asuntos en los documentos de cúpula. Se observa también el aumento de otros temas que tratar. Tal evolución va acompañada de una estrategia clara del grupo: ampliar las discusiones que tienen que ver con intereses convergentes, pero evitando fricciones. Si no se discuten temas que ocasionen divergencias geopolíticas, el grupo fortalece su actuación y sus relaciones. ${ }^{35}$

Para hacer una comparación, el primer documento conjunto elaborado (Declaración de Ecaterimburgo, 2009) se limitó a 16 temas centrados en aspectos económicos, financieros y de fomento a la cooperación. Sin embargo, en la última declaración de cúpula aquí estudiada (Declaración de Ufá, 2015) se presentaron más de cinco temas en comparación con la primera declaración (véase cuadro 1). Este hecho reveló no sólo el aumento de la agenda, sino también de sus objetivos. La VII Declaración de cúpula de los BRICS, a pesar de estar dirigida a los temas económicos y financieros predominantemente, trató temas diversos como derechos humanos, desarrollo sustentable y hasta de seguridad -el que se tratará en la siguiente sección.

Después de un breve periodo considerado unipolar -con la preponderancia de Estados Unidos en la definición de las reglas internacionales- y el surgimiento de un orden marcado por la "ascensión del resto", 36 se entiende que, según sus capacidades, los estados emergentes procuran obtener un papel más activo en la gobernanza internacional, lo que puede comprobarse, como se comentó, por el aumento cuantitativo de las declaraciones y de los temas abordados en las siete reuniones aquí presentadas. Como lo señala Fonseca Jr. en su artículo citado, "en el marco de este amplio proceso, la transformación del BRICs en una instancia política, aunque informal, establece que, si separados ya eran una influencia,

${ }^{35}$ A. F. Cooper y A. B. Farooq, "BRICs and the Privileging of Informally in Global Governance”, Global Policy, vol. 4, núm. 4, 2013, pp. 428-432.

${ }^{36}$ Zakaria, op. cit. 
juntos pueden ser de mayor influencia”. En este sentido, la evolución de los BRIcs tiene una función relevante para entender el orden internacional, porque como grupo estaría en la raíz de la multipolarización de ese orden (p. 18).

Si bien constituido con cierta informalidad, las recientes articulaciones internas muestran importancia significativa en la gobernanza entre los estados, donde

las reuniones regulares de autoridades (de alto nivel) ya significan ganancia para los cinco en la medida en que encuentra un foro privilegiado y exclusivo para intercambiar información sobre la situación regional y mundial. El mismo hecho de tener peso regional y participación (diferenciada) en los temas globales puede estimular y enriquecer el diálogo (ibid.).

Las articulaciones en el grupo se reflejan con claridad en la agenda internacional, sobre todo en asuntos comerciales y financieros. Algunos autores argumentan la manifestación de una característica más ambiciosa en el ámbito del orden internacional: mover la balanza del poderío de Estados Unidos y de sus aliados de Occidente (Unión Europea y Canadá):

El objetivo es encadenar a Gúliver de todas las formas posibles sin importar lo finas que puedan ser las amarras. No sorprende, entonces, que Brasil e India sean el cuarto y quinto país que reclamen más activamente sobre el mecanismo para solucionar las controversias de la oмc. Tampoco deja de sorprender que Brasil, China e India quieran usar las instituciones internacionales para resistir los intentos de Norteamérica para promover nuevas normas en el uso de la fuerza, cuestionar el principio de soberanía, usar la fuerza para obligar a cambiar el régimen. ${ }^{37}$

Fonseca Jr. denomina este movimiento como self-balancing. ${ }^{38}$ Esa idea, entonces, llevaría al BRICs a buscar la administración de los

${ }^{37}$ Hurrell, "Hegemonia, liberalismo e ordem global...", p. 27.

38 Art. cit., p. 23. 
asuntos más sensibles del orden internacional. Lo que parece evidente, si se entiende que, según el grupo se fortalezca, otros asuntos -y no puramente económicos o financieros- se integrarán a la agenda, ${ }^{39}$ en especial los relativos a la seguridad internacional.

En la última sección del artículo se intenta identificar que en las declaraciones de la cúpula de los BRICs aparezcan los asuntos sobre seguridad, por ser uno de los documentos más importantes de la agenda general conjunta del grupo y por la que el grupo se manifiesta. Este hecho comprobado va de la mano con la idea de que esos estados no ignoran que, agrupados, pueden influir substancialmente en la gobernanza global, en especial en los asuntos más sensibles, como la seguridad.

\section{LOS BRICS Y LA SEGURIDAD INTERNACIONAL}

Esta sección procura enfrentar el problema central del objeto de estudio: el análisis de la inserción de los temas de seguridad internacional en la agenda de los BRICs. En ese sentido, se resalta el uso de la perspectiva negativa de la seguridad. Según Gjorv, seguridad negativa ${ }^{40}$ es la que

[está] asociada con la seguridad tradicional, fundamentada en premisas sobre cuestiones de seguridad y de Estado definidas universalmente, dirigidas por un acuerdo universal sobre la herramienta de seguridad -la fuerza militar [...]. Seguridad negativa emplea una epistemología del miedo, enfocada en la identificación de amenazas y el uso de la violencia. ${ }^{41}$

${ }^{39}$ Véase Reis, art. cit.

${ }^{40}$ La idea de seguridad negativa se desprende de dos estudios tradicionales de la EI, en especial de la obra de Bull, ya citada. Para una lectura más profunda sobre el debate que abarca el concepto de seguridad, véanse D. Baldwin, "The Concept of Security”, Review of International Studies, núm. 23, 1997, pp. 5-26, y B. Bazan, People, states and fear: An Agenda for security Analysis in the PostCold War Era, Brighton, Weatsheaf, 1991.

41 "Security by any other name: negative security, positive security, and a multi-actor security approach”, Review of International Studies, vol. 38, núm. 4, 2012, pp. 836-839. 
Antes de entrar en la discusión central es importante identificar, sin embargo, la posición en que se encuentran los países en el mapa de la seguridad. En tales términos, el grupo está formado por miembros bastante dispares. En el mapa se identifican tres potencias nucleares y dos miembros permanentes del Consejo de Seguridad de las Naciones Unidas (CSNU). Así, China y Rusia están mejor posicionadas, porque cuentan con armas nucleares y son miembros permanentes del cSNU. La India, Brasil y África del Sur siguen en la contienda por un asiento permanente en el Consejo de las Naciones Unidas. India desarrolló recientemente armas nucleares. Brasil y África del Sur llevan relativa desventaja en ese sentido, ya que ambos son signatarios del Tratado de la no proliferación de armas nucleares (Tratado de Tlatelolco).

En el cuadro 1 (cf. supra) se muestra que los BRIcs llevaron a cabo siete cúpulas entre 2009 y 2011, con la presencia de los jefes de Estado, en las que se trataron problemas de seguridad de modo directo o tangencial. Por los análisis de las relatorías de cúpula, ${ }^{42}$ se comprobó la concurrencia de la temática, es decir el foco central de análisis de esta última sección. Es importante resaltar que el uso de una única fuente de información puede traer limitaciones para el análisis mostrado. Es más: puede darse, porque las relatorías de cúpula son los únicos documentos generales publicados sistemáticamente por los BRICs.

Para identificar la densidad de los temas de seguridad en las cúpulas, fue necesario establecer categorías textuales representadas por palabras-clave. La primera categoría es la de seguridad, seleccionada por su referencia directa al objeto del artículo, cuya representación se da por la palabra-clave "seguridad".

La segunda categoría es paz, porque indica la voluntad de los BRICS de posicionarse en la solución de conflictos, tensiones o desequilibrios internacionales. Esta categoría se identifica por las palabras-clave "paz”, "pacíficamente” y "pacificar”. La última categoría

${ }^{42}$ Las relatorías de cúpula o declaraciones de cúpula son documentos publicados después de cada encuentro anual de los BRICs que refieren a los principales asuntos discutidos por los países miembros. En ellos se exponen las intenciones y principios que guían las acciones del grupo y van suscritos por los jefes de Estado de Brasil, Rusia, India, China y África del Sur. 
es terrorismo, que se identifica por las palabras-clave "terrorismo", "terror" y "terrorista", aquí establecida por su importante influencia en definir la agenda de seguridad global y porque Rusia, India y China sufren de problemas internos de terrorismo.

Mediante un software ${ }^{43}$ específico para contar la frecuencia de cada palabra en un texto determinado, se elaboró el cuadro 2. Dada la cantidad en el uso de la palabra seguridad y su significado polisémico, fue necesario llevar a cabo un análisis cuidadoso de su ocurrencia en las relatorías de las cúpulas. Además del uso del software para el conteo de las palabras, también se comprobó la conexión de la palabra-clave con otros sinónimos para garantizar la cuantificación precisa del objeto del estudio.

El análisis primario del texto referente a la cúpula de 2009 identificó la ocurrencia de la palabra "seguridad"; doce relacionadas con la seguridad alimentaria; una, con la seguridad energética, y una, con la seguridad de las rutas de energía. Sin embargo, el cuadro 1 marca cero menciones, toda vez que ninguna de las ocurrencias se refiere a la problemática del artículo, por lo que los datos desprendidos en el total de las relatorías de las cúpulas se tomaron como un análisis cualitativo de apoyo. El cuadro 2 presenta la consolidación de los datos, descartando las ocurrencias de la palabra-clave "seguridad" no relacionadas con el objetivo de estudio.

Revisando las ocurrencias de las palabras-clave, se observa el aumento del tema en las cúpulas de manera general. Situación que se hace más evidente cuando se analiza que la categoría "seguridad" observa un crecimiento significativo a lo largo de las reuniones. El término se mostró de 0 a 43 ocurrencias, señal del aumento de importancia del tema para el grupo.

La segunda categoría analizada ( $p a z)$ muestra un aumento de ocurrencia en el recorrer de las cúpulas. Si bien la tendencia no es consistente, hay una oscilación entre la primera y la segunda reunión. En las cúpulas de 2011, 2012, 2013 y 2014, se observa el aumento de la ocurrencia del término en los documentos, semejante al número de apariciones de la palabra-clave "seguridad".

${ }^{43}$ Se utilizó la combinación de software R, versión 3.1.0, y el suplemento Iramuteq para el análisis del léxico contenido en los documentos atinentes a las cúpulas. 
Cuadro 2

Ocurrencia de las palabras-clave por documento final, por cúpula

\begin{tabular}{|c|c|c|c|}
\hline $\begin{array}{c}\text { Palabras-clave } \\
\text { Cúpula }\end{array}$ & Seguridad $^{\mathrm{a}}$ & $\begin{array}{l}\text { Paz/pacificar/ } \\
\text { pacificamente }\end{array}$ & Terrorismo/terror/terrorista \\
\hline I cúpula (2009) & 0 & 2 & 3 \\
\hline II cúpula (2010) & 2 & 1 & 9 \\
\hline III cúpula (2011) & 8 & 5 & 4 \\
\hline IV cúpula (2012) & 9 & 8 & 5 \\
\hline V cúpula (2013) & 15 & 15 & 7 \\
\hline VI cúpula (2014) & 25 & 29 & 17 \\
\hline VII cúpula (2015) & 43 & 25 & 28 \\
\hline
\end{tabular}

a Las cantidades de ocurrencia de la palabra-clave "seguridad" aquí presentadas se han ajustado para reflejar únicamente las veces que el término se refirió al objeto de este estudio.

Respecto a "terrorismo/terror/terrorista", se observa también consistencia en cuanto a su aumento en los textos. Es importante apuntar que la categoría textual terrorismo está presente en todos los documentos, indicador de la preocupación constante del tema, si bien en grado distinto, en todas las reuniones.

Además de contar las palabras-clave en las relatorías de cúpula, se hizo también un análisis de la cantidad de puntos en que se mencionaron los léxicos de interés del estudio. El cuadro 3 muestra los puntos en que "seguridad", "paz/pacíficamente/pacificar" y "terrorismo/terror/terrorista" aparecen. Es importante destacar que el cuadro 3 no muestra información del número de puntos dedicados integralmente al tema, pero lo hace en la cantidad de puntos en que apareció cada palabra-clave. De ahí que debe considerarse que un mismo punto puede contener más de una palabra-clave.

El análisis del léxico es un auxiliar para estudiar el panorama general de la seguridad entre los miembros del BRICs, porque presenta el comportamiento del tema durante las diversas reuniones. Arroja las pistas de posibles modificaciones en los objetivos del grupo, evidencia su evolución, que se deriva de un arreglo mayoritariamente económico preocupado por la estructura financiera mundial y comienza a incorporar temas de la llamada high politics. Como lo 
muestra el cuadro 3, en la primera Declaración de cúpula, los temas de seguridad apenas se ocuparon en 3 (12.5\%) de los 16 puntos tratados; en 2015 fueron 28 puntos (36.4\%) dedicados parcial o integralmente a cuestiones de seguridad, en un total de 77 .

\section{Cuadro 3}

Cantidad total de puntos versus puntos que tratan las palabras-clave

\begin{tabular}{|c|c|c|c|c|c|c|}
\hline $\begin{array}{c}\text { Año } \\
\text { de la } \\
\text { cúpula }\end{array}$ & $\begin{array}{l}\text { Total de } \\
\text { puntos } \\
\text { en la } \\
\text { relatoría } \\
\text { de cúpula }\end{array}$ & $\begin{array}{l}\text { Puntos de } \\
\text { relatoría de } \\
\text { cúpula que } \\
\text { contienen } \\
\text { la categoría } \\
\text { seguridad }\end{array}$ & $\begin{array}{l}\text { Puntos de } \\
\text { relatoría de } \\
\text { cúpula que } \\
\text { contienen la } \\
\text { categoría paz }\end{array}$ & $\begin{array}{l}\text { Puntos de } \\
\text { relatoría de } \\
\text { cúpula que } \\
\text { contienen } \\
\text { la categoría } \\
\text { terrorismo }\end{array}$ & $\begin{array}{l}\text { Puntos de } \\
\text { relatoría de } \\
\text { cúpula que } \\
\text { versan sobre } \\
\text { cuestiones de } \\
\text { seguridad }\end{array}$ & $\begin{array}{c}\% \text { de } \\
\text { puntos con } \\
\text { temas de } \\
\text { seguridad }\end{array}$ \\
\hline 2009 & 16 & 0 & 2 & 1 & 2 & $12.5 \%$ \\
\hline 2010 & 31 & 1 & 1 & 2 & 4 & $13 \%$ \\
\hline 2011 & 32 & 6 & 4 & 1 & 7 & $22 \%$ \\
\hline 2012 & 50 & 8 & 7 & 2 & 12 & $24 \%$ \\
\hline 2013 & 47 & 8 & 8 & 2 & 12 & $25.5 \%$ \\
\hline 2014 & 72 & 14 & 15 & 5 & 21 & $29.2 \%$ \\
\hline 2015 & 77 & 23 & 15 & 9 & 28 & $36.4 \%$ \\
\hline
\end{tabular}

Fuente: elaboración de los autores.

Identificar la presencia de las palabras-clave y el mapeo de los puntos es importante para visualizar los cambios en el grupo, por lo que por ahora resulta una herramienta inicial y limitada. Es necesario profundizar en la evaluación de los textos para sacar a la superficie las preocupaciones reales de los BRICs sobre seguridad, por lo que desmenuzar el contenido de las cúpulas es una tarea primordial para proporcionar detalles sobre la importancia de la seguridad para el grupo.

En la I cúpula llevada a cabo en 2009, todavía con el signo de BRIC (sin África del Sur), el tema de seguridad prácticamente no estaba contemplado. El documento se concentra en cuestiones relacionadas con modificaciones de la estructura macroeconómica mundial y la preocupación por la seguridad alimentaria, en especial en 
el mundo en desarrollo. De un total de los 16 puntos, apenas dos (puntos 12 y 13) mencionaron tangencialmente las cuestiones sobre seguridad. El documento destaca el compromiso del grupo por "resolver pacíficamente la controversia en las relaciones internacionales". ${ }^{44}$ Además, reafirma su repudio a cualquier forma de terrorismo y a los actos que perjudiquen la paz, el equilibrio y la armonía internacionales.

El grupo reconoció en 2010 los cambios por los que ha pasado el orden internacional y se posicionó como actor de tal cambio. En ese sentido, el BRIC comparte "la idea de que el mundo está experimentando cambios importantes y rápidos que realzan la necesidad de transformaciones correspondientes a la gobernanza global en todas las áreas relevantes", ${ }^{45}$ manifestando la opinión de que el grupo tiene legitimidad y capacidad para participar de esa reformulación, como lo señaló Hurrell (véase la primera sección).

El grupo expresa su convicción en el diálogo y en la cooperación como mecanismo para crear un mundo pacífico y armónico, en el que "los países del BRIC [propicien] no sólo [...] servir a los intereses comunes de las economías emergentes de mercado y de los países en desarrollo, sino también en la construcción de un mundo armonioso, de paz duradera y prosperidad común". ${ }^{46}$

La importancia del Derecho Internacional (DI) y de la diplomacia multilateral son premisas básicas manifestadas en todas las relatorías de cúpula. El BRIcs expresa que el deseo del grupo es por la "paz, seguridad, desarrollo y cooperación". ${ }^{47}$ La cúpula de 2011 -primera con la presencia de África del Sur- reitera la importancia de los BRICs en la gobernanza internacional, ámbito global en el que han desempeñado un papel central, así como

${ }^{44}$ I Declaración de cúpula del BRIC, 2009, en http//brics.itamaraty.gov.br/ pt_br/categoría-portugues/20-documentos/73-primeiro-declaracao

45 II Declaración de cúpula del BRIC, 2010, en http//brics.itamaraty.gov.br/ pt_br/categoría-portugues/20-documentos/74-segunda-declaracao-conjunta

${ }^{46}$ Idem.

${ }^{47}$ III Declaración de cúpula del BRICs, 2011, en http//www.itamaraty.gov.br/ pt-BR/notas-a-imprensa/2559-iii-cupula-do-brics-sanya-14-de-abril-de-2011 
"contribuido de manera significativa para la paz mundial, la seguridad y la estabilidad". 48

La IV cúpula de 2012 fue el marco para que el grupo tratara los problemas de seguridad. En la reunión, este asunto se consideró, por primera vez, como el tema de la cúpula, intitulada "BRICS - Asociación para la estabilidad global, seguridad y prosperidad". De un total de 50 temas, diez de ellos se destinaron, directa o indirectamente, al tema. Se resaltó la cooperación y el diálogo promovido por el grupo para impulsar la seguridad y la paz, destacando el hecho de que los BRICs incluyen países de diversas regiones del mundo (Asia, América Latina, África y Europa).

Durante las cúpulas del BRICs, se destacan algunos temas sobre seguridad, entre otros, como de extrema importancia para el grupo, sea por la cantidad de veces sumadas en que aparecen o por el lugar de privilegio en la agenda de seguridad internacional. En ese sentido, el terrorismo, la reforma del CSNU, la inestabilidad en Oriente Medio y en el Norte de África y el narcotráfico son temas de la mayor relevancia y, por lo mismo, se tratarán a lo largo de esta sección.

El terrorismo adquiere importancia a partir de la II cúpula (2010). El todavía BRIC llamaba la atención para que se cumplieran los diversos tratados establecidos en el ámbito de la ONU, además de solicitar redoblar esfuerzos para la aprobación de la Convención Global sobre Terrorismo Internacional (CGTI). En 2011, los BRICS condenaron la práctica de ataques terroristas y apoyaron la creación de medidas preventivas y punitivas en el combate a ese tipo de práctica, reiterando la necesidad de aprobar la CGTI en el ámbito de la ONU. De manera general, el CGTI se ve como el principal instrumento normativo con fuerza suficiente para lidiar con el grave problema del terrorismo, razón por la cual todas las cúpulas han reforzado la importancia de su aprobación.

En el tratamiento del terrorismo, la III cúpula fue la primera en mencionar el crimen cibernético como un instrumento terrorista. Es clara la postura del BRICs en cuanto a su repudio a cualquier 
forma de terrorismo y se coloca como agente de cooperación para enfrentar este problema, entendido como un "flagelo internacional". ${ }^{49}$ En 2015, durante la VII cúpula del BRICs, llevada a cabo en Rusia, ${ }^{50}$ el tema del terrorismo ganó importancia en lo tocante a la cooperación del grupo con el Combate al lavado de dinero y al Financiamiento del terrorismo y proliferación, del Grupo de Acción Financiera (GAFI). Por primera vez, además, el grupo señaló el extremismo y fundamentalismo como la raíz del terrorismo internacional.

El terrorismo adquirió importancia en la agenda de la seguridad internacional después de los ataques del 11 de septiembre de 2001, de modo tal que el interés del BRIcs dedicado a este tema es, en parte, consecuencia de los problemas internos de los países, en especial China, India y Rusia, que combaten grupos terroristas. Por ejemplo, en 2010, India (atentado en Pune ${ }^{51}$ ) y Rusia (atentado en el metro de Moscú ${ }^{52}$ ) sufrieron ataques terroristas en sus territorios.

Las cúpulas reforzaron a partir de 2010 las aspiraciones del grupo a reorganizar las instituciones que cuidan la seguridad internacional, en especial el cSNU. Sin dejar de reconocer la importancia de la onU como la institución responsable por la regulación de los desafíos y amenazas globales, el BRICs tiene deseos de influir en la reforma de los arreglos sobre seguridad de la onU, con la intención de "asegurar mayor eficacia, eficiencia y representativi-

${ }^{49} I d$.

${ }^{50}$ VII Declaración de cúpula del BRICS, 2015, en http//www.itamaraty.gov. $\mathrm{br} / \mathrm{pt}-\mathrm{BR} /$ notas-a-imprensa/10465-vii-cupula-do-brics-declaracao-de-ufa-ufa-russia-9-de-julho-de-2015

${ }^{51}$ Ocurrió en 2010 en la ciudad de Pune, Maharashtra, India. Un grupo islámico extremista hizo explotar una bomba junto a una panadería italiana en el centro de la ciudad. En el atentado murieron diecisiete personas y más de cincuenta resultaron heridas (USCIRF, "Annual Report of the United States Commission on International Religious Freedom”, en http://www.uscirf.gov/sites/default/files/ resources/annual1\%20report\%202010.pdf).

${ }^{52}$ El 30 de marzo de 2010 dos mujeres bomba detonaron explosivos en el metro de Moscú; treinta y ocho personas perdieron la vida. Véase http:/www.wahsingtonpost.com/wp- 
dad del modo en que se puedan enfrentar de mejor manera los desafíos globales de la actualidad" ${ }^{53}$

La posición oficial del BRICs sostiene que países como Brasil, India y África del Sur merecen mayor protagonismo en las Naciones Unidas y, en particular, en el Consejo de Seguridad. La cúpula de 2011 destacó la participación de los países integrantes del BRICS, porque ofrecieron una "valiosa oportunidad de trabajar de forma conjunta y estrecha en los problemas de paz y seguridad, para reforzar los puntos de vista multilaterales y facilitar la futura coordinación sobre los temas de la agenda del CSNU". ${ }^{54}$

El Consejo de Seguridad es el órgano por excelencia que revela las desigualdades de poder del sistema internacional señaladas por Bull, reivindicación que muestra, por un lado, la opinión de que el arreglo actual no ha contribuido a una mejor distribución del poder vigente $y$, por otro, que los estados del grupo que no son miembros permanentes tengan un lugar legítimo en la nueva estructura que se pretende defender. Sin embargo, y a pesar de la postura favorable respecto a la reforma del CSNU, se trata de uno de los temas más complejos del grupo. Brasil y la India forman parte del llamado G-4 (conjuntamente con Japón y Alemania), ${ }^{55}$ grupo que favorece la reforma del Consejo y su consecuente inserción como miembros permanentes. Por su parte, China aún se resiste a esa reformulación, en especial por su rivalidad con India, lo que termina siendo un problema serio que puede afectar las pretensiones de la actuación concertada del grupo. ${ }^{56}$

53 III Declaración de cúpula del BRICS, 2011, citada.

${ }^{54} \mathrm{Id}$.

${ }^{55}$ En 2015, se reunieron los miembros del G-4 en Nueva York para discutir la reforma del cSNU. El acta del encuentro está disponible en http://www.mofa.go. jp/files/000101241.pdf

${ }^{56}$ Para Z. Laidi (“BRICS: Sovereignty power and weaknes", International Politics, vol. 49, núm. 5, 2012, p. 621), China es uno de los estados más resistentes a la democratización del sistema internacional cuando los asuntos van más allá de la ampliación de sus propios derechos; el caso de la reforma del Consejo de Seguridad es uno de los más paradigmáticos. Según el autor, "el BRICs termina siendo una coalición de las más curiosas, en que sus miembros buscan neutralizarse unos a otros en las áreas más estratégicas del poder". 
La III cúpula se llevó a cabo en un momento turbulento en el Oriente Medio y el Norte de África, porque se observó la escalada de las tensiones de la Primavera Árabe. La estabilidad de esas regiones ha sido un tema importante en las cúpulas desde 2011. El BRICS resalta la importancia de los organismos regionales para poner fin a las tensiones en el Oriente Medio y en el Norte de África, ratificando su apoyo a las acciones del Panel de alto nivel de la Unión Africana. ${ }^{57}$

La crisis de Libia también fue estudiada sobre todo por el rechazo de las acciones de la Organización del Tratado del Atlántico Norte (OTAN). Es importante señalar que la resolución de la ONU para Libia se expidió el 17 de marzo de 2011, con el único voto favorable de África el Sur sobre la abstención de los demás miembros de los BRICs. ${ }^{58}$

En la IV cúpula, Siria ${ }^{59}$ significó la preocupación central por su relación directa con el caso de Libia. Se pidió poner fin a la violencia y a las violaciones de los derechos humanos y, al mismo tiempo, se solicitó el "respeto a la independencia y a la integridad

${ }^{57}$ Se puede afirmar de manera general que se establece un Panel de alto nivel con el fin de "recomendar medidas claras y prácticas para asegurar una efectiva acción colectiva en la promoción de la paz y la seguridad nacional” (J. C. Baena Soares, "As Naçoes Unidas diante das Ameaças, dos desafios, das mudanças", CEBRI Dossiê, vol. 1, año 4, 2005, p. 6). En el ámbito de la Unión Africana, como ejemplos recientes, se tiene High Level Panel on Darfur, High Level Panel Duscussion on African Climate Solutions; y High Level Implementation Panel (sobre las negociaciones que cuidan la independencia de Sudán del Sur).

${ }^{58}$ Véase "Asian Perspectives on Humanitarian Intervention in the $21^{\text {st }}$ Century", Doshisha 2nd International Conference on Humanitaria Intervention, 2012, en http://gdoshisha.files.wordpress.com/2012/06/sridhar-patnaik-pa per.pdf

${ }^{59} \mathrm{El} 4$ de noviembre de 2011, el CNSU intentó votar una resolución para el caso sirio. Rusia y China, miembros permanentes, usaron su poder de veto, mientras que Brasil, India y África del Sur, miembros no permanentes en ese momento, se abstuvieron. Para mayor detalles de la votación del BRICs, véase L. Pérez y M. Brito, "Os bRICs na Votação do Projeto Europeu de Resolução do Conselho de Segurança Sobre a Síria”, BRICs Policy Center - BRICs Monitor, noviembre de 2011, en http:/ / bricspolicycenter.org/homolog/publicacoes/Interna/881?tipo=Brics\%20Monitor, consultado el 4.I.2017. 
territorial y a la soberanía [de Siria]"60, como una clara oposición a las acciones de la OTAN emprendidas en 2011. Es evidente, en ese caso, que la visión de los BRICs centrada en el estado, sobre todo por el hecho de cuestiones de "soberanía nacional en sentido estricto, no puede ser una fuente de discusión en el ámbito de los proyectos políticos colectivos". ${ }^{61}$

Lo que incomodó -e incomoda- particularmente a los estados del grupo en los dos casos, es la posibilidad del uso de la fuerza para los intereses de las potencias occidentales, en especial lo relativo al cambio de régimen. Tal fue la justificación central para que Rusia y China vetaran las propuestas de Siria $^{62}$ de intervención. Sin embargo, el posicionamiento de los estados miembros revela que esa postura dice más respecto al debate que a la forma de intervención y mucho menos que la legitimidad. ${ }^{63}$ Brasil presentó una propuesta al respecto con el fin de establecer criterios en una intervención, en el documento llamado Responsabilidad de proteger. ${ }^{64}$ Recientemente, China ha intentado debatir tal cuestión bajo

${ }^{60}$ IV Declaración de cúpula del BRIcs, 2012, en http://brics.itamaraty.gov. br/pt_br/categoria-portugues/20-documentos/76-quarta-declaracao-conjunta

${ }^{61}$ Laidi, art. cit., p. 623.

${ }^{62}$ Fueron tres vetos de China y Rusia, seguidos de abstenciones de Brasil, India y África del Sur. Sobre el caso, véase R. Thakur, "R2P after Libya and Syria: Engaging Emerging Powers”, The Washington Quarterly, vol. 36, núm. 2, 2013, pp. 61-76.

63 Tal es el argumento del diplomático canadiense Gareth Evans, uno de los creadores de la Responsabilidad de proteger. En entrevista, Evans afirmó que la contestación de los BRICS en lo tocante a la intervención en Libia no fue por el inicio de la acción militar, sino por una política de cambio de régimen establecida por Estados Unidos, Francia y Gran Bretaña durante la intervención. Véase http:/ / www.globalr2p.org/media/files/gareth-evans-on-responsibility-to-protect-after-li bya.pdf

${ }^{64}$ El documento de Brasil fue una contribución a los debates sobre la Responsabilidad de proteger, instituto promovido a raíz de un documento de la International Commission on Intervention and State Sovereingty en 2001, que busca asociar la soberanía a la responsabilidad. Según el documento, en casos graves de violaciones, estados negligentes pueden sufrir la intervención militar externa. En 2005, la R2P quedó institucionalizada en la onU durante la World Summit, únicamente para casos de genocidio, limpieza étnica, crímenes de guerra y crímenes contra la humanidad. 
una noción titulada Responsible Protection. ${ }^{65} \mathrm{El}$ hecho revela la pretensión de esos Estados de tener un papel más activo en la regulación de un asunto tan sensible para la seguridad internacional como lo es una intervención militar externa.

De forma adicional, la cuestión de Siria revela una clara contraposición en los intereses de los países Occidentales -en especial EUA, Francia y Reino Unido- y el apoyo histórico de Rusia al régimen de Siria y al presidente Bashar al-Assad. ${ }^{66}$ La reafirmación de la soberanía siria se ha mencionado con frecuencia en las declaraciones. La V cúpula (2013), por ejemplo, resaltó la oposición del grupo a cualquier acción militar externa sobre Siria, destacando el respeto por la independencia, soberanía e integridad del Estado.

En relación con la preocupación de la inestabilidad en el Oriente Medio, el BRIcs resaltó el carácter fundamental de adoptar medidas definitivas para finalizar las tensiones interminables, como lo es el conflicto árabe-israelí. En 2013, el BRIcs llamó la atención a las violaciones del Di por la construcción de asentamientos israelíes y convocó a la comunidad internacional a ayudar a los israelíes y palestinos a establecer un Estado Palestino "contiguo y económicamente viable". ${ }^{67}$

El BRICs está preocupado por la seguridad de Afganistán e Irán. A Irán se le ha delegado el papel central en la paz del Oriente Medio. Sin embargo, el programa nuclear del país se aprecia como una posible amenaza en caso de no desarrollarse dentro de las estrictas directrices de pacifistas. Para continuar con el programa se requiere de un diálogo continuo con la Agencia Internacional de Energía Atómica (AIEA). ${ }^{68}$

${ }^{65}$ A. Garwood-Gowers, “China's «Responsible Protection» Concept: Reinterpreting the Responsibility to Protect (R2P) and Military Intervention for Humanitarian Purposes", Asian Journal of International Law, vol. 6, núm. 1, 2016, pp. 89-116.

${ }^{66} \mathrm{R}$. Thakur, art. cit.

${ }^{67}$ V Declaración de cúpula del BRICs, 2013, en http:/ / brics.itamaraty.gov.br/ pt_br/sobre-o-brics/desenvolvimentos-recentes-v-cupula-durban-africa-do-sul27-de-marco-de-2013

${ }^{68}$ Herz y Coutinho (art. cit., p. 44) recuerdan que los estados del grupo han apoyado las ideas de la no proliferación y desarme, "por lo que discrepan en cuanto al proceso de reforma y fortalecimiento del régimen [... y sobre la crítica a la clasificación de países nucleares y no nucleares". 
Con respecto de Afganistán, el grupo lo tiene por foco de inestabilidad en el Oriente Medio. El BRICs destaca su compromiso en "apoyar el surgimiento de Afganistán como un Estado pacífico, estable y democrático, libre del terrorismo y del extremismo". ${ }^{69}$ Además, el grupo señala "la necesidad de una cooperación regional e internacional más eficaz". ${ }^{70}$

El resultado más importante de la VI cúpula llevada a cabo en $2014^{71}$ en Brasil fue la creación del Banco de los BRICs. Sin embargo, la seguridad también fue importante en la relatoría final del grupo, presente en 14 de los 72 puntos de la Declaración de Fortaleza. Además del reclamo de paz y reforma del csNu, la inestabilidad en Oriente Medio y Afganistán dieron la pauta. La novedad del documento fue condenar el fortalecimiento de la seguridad de un país en detrimento de la seguridad de los demás, en una alusión a la inestabilidad en Europa por la crisis de Ucrania, en la que Rusia tiene intereses directos.

La cúpula de 2014 toca, por primera vez, el tema del tráfico de drogas. Es importante señalar que el grupo no considera este asunto como problema de seguridad internacional, pero intenta tratarlo como problema mundial de salud y seguridad pública, situación contraria al modelo predominante de enfrentamiento al narcotráfico. En la declaración de 2015, el documento significaba el apoyo a las conclusiones del Plan Global de Acción Conjunta (JCPA), que permitiría a Irán ejercer su soberanía en el desarrollo de su programa nuclear de forma pacífica.

En las cúpulas se trataron temas de seguridad, pero también se llevaron a cabo otras reuniones relativas al problema. Entre 2009 y 2013, se efectuaron seis encuentros entre encumbrados funcionarios $^{72}$ para tratar la seguridad de manera general, o bien sobre un tema específico:

${ }^{69}$ IV Declaración de cúpula del BRICS, 2012, citada.

${ }^{70} \mathrm{Id}$.

${ }^{71}$ VI Declaración de cúpula del BRICs, 2014, en http://brics.itamaraty.gov. $\mathrm{br} / \mathrm{pt}$ _br/categoria-portugues/20-documentos/224-vi-cupula-declaracao-e-planode-acao-de-fortaleza

72 Dentro de los documentos no se encontraron definiciones de "encumbrados funcionarios", como tampoco indicación de sus niveles jerárquicos. No se divulgaron las agendas ni las relatorías de reunión. 
a) Reunión de altos funcionarios responsables por temas de seguridad (Moscú, Rusia, del 28 al 30 de mayo);

b) Reunión del BRIC al margen del Encuentro internacional de funcionarios de alto nivel responsables por asuntos de seguridad (Sochi, Rusia, 5 y 6 de octubre);

c) Reunión de altos funcionarios responsables por temas de seguridad (Brasilia, Brasil, 15 de abril);

d) Reunión de viceministros de relaciones exteriores del BRICS sobre la situación en el Oriente Medio y el Norte de África (Moscú, Rusia, 24 de noviembre);

e) Reunión BRICs al margen del III Encuentro internacional de altos funcionarios responsables por temas de seguridad (del 6 al 8 de junio, en San Petesburgo, Rusia);

f) Reunión de altos funcionarios responsables por temas de seguridad y estrategia (Nueva Deli, India, 10 y 11 de enero).

Si bien los BRICs llevaron a cabo encuentros regulares para tratar específicamente los asuntos de seguridad, no se produjo ningún documento derivado, como tampoco se divulgó la agenda de los temas tratados en cada encuentro, situación que dificulta un análisis profundo del pensamiento conjunto sobre seguridad. Pero la mera existencia de esas reuniones refuerza la perspectiva de que las cuestiones de seguridad están ganando espacio cada vez más en el BRICS y, por consiguiente, tienen un efecto en la actuación del grupo en temas duros del orden internacional.

En esta sección hay evidencias del crecimiento cuantitativo y cualitativo de los asuntos sobre seguridad en el ámbito de las declaraciones de cúpula más recientes de los BRICs, si se las compara con las primeras. El trato cada vez más frecuente en esos documentos de asuntos delicados de la política internacional, demuestra que hay una sólida búsqueda por la afirmación del grupo, ya que se manifiesta a favor de la agenda internacional sobre seguridad.

Este hecho constituye un reconocimiento buscado que, como se ha visto a lo largo del documento, es parte de la dinámica política mundial que prevalece en un ambiente de jerarquías. ${ }^{73}$ Hay indi-

${ }^{73}$ Véase Hurrell, "Hegemonia, liberalismo e ordem global...". 
cios significativos, además, para reconocer al BRICS como una pieza central en la gobernanza global, porque ser parte de la regulación de tales cuestiones -como se infiere de la teoría de la EI- es prerrogativa de las grandes potencias. ${ }^{74}$

\section{CONSIDERACIONES FINALES}

La EI de RI sostiene el concepto de que, en el ambiente internacional, hay una sociedad de estados que navega entre un sistema anárquico y una comunidad internacional, escenario que se asemeja a un ambiente de coordinación en que los estados buscan administrar sus divergencias mediante la satisfacción de valores e intereses comunes. Por medio de una estructura societaria creada, los estados establecen una gobernanza centrada en normas y reglas, así como en instituciones -sean éstas formales o informales.

En el centro de esta gobernanza, mantener el orden internacional es uno de los asuntos de mayor importancia y, dentro de ese orden, la seguridad es una preocupación fundamental. Eso se da porque en esa estructura de sociedad anárquica los valores que predominan son los de coexistencia y de sobrevivencia. De tal modo que las normas y reglas que deben lidiar con los asuntos de seguridad se colocan en primer plano y, por eso, están limitadas a una gestión restringida a pocos estados.

Bull, en el trabajo que hemos venido citando, nos recuerda que los principales actores y responsables del orden internacional y, en consecuencia, de las reglas que rodean a los asuntos de seguridad son las grandes potencias. En un breve periodo durante la post-Guerra Fría, se identificó a Estados Unidos como el gran norm setter, es decir el indicativo de la existencia de un momento unipolar. Sin embargo, ese factor ha cambiado en las dos últimas décadas.

Las potencias emergentes están desafiando cada vez más a Estados Unidos -acompañado por sus aliados-, si bien todavía de forma benigna. Pero en ese sentido llaman la atención algunos

${ }^{74}$ Véase Bull, op. cit. 
hechos: el primero, el ascenso de China y el segundo, la recuperación de Rusia. Esas dos potencias importantes (conocidas como G-2), con la alianza de las llamadas potencias regionales emergentes (Brasil, India y África del Sur), han establecido nuevos desafíos en cuanto al tema de la gobernanza internacional en la post-Guerra Fría. No cabe duda de que esos estados, por sí mismos, y teniendo a la vista los poderes relativos que detentan, podrían ser piezas importantes en la gobernanza internacional. ${ }^{75}$ Pero el principio de desafío aquí estudiado se da no sólo en sentido de ser un polo importante, sino como la idea de que el grupo puede ser una pieza central -al menos en cuestiones mediadas conjuntamente.

Cabe señalar que no interesa en este estudio resolver el anhelo de los BRICs sobre la gobernanza internacional, sobre todo en lo que se refiere a la seguridad, ni mostrar indicios sobre cómo sería un futuro (y, sobre todo, incierto) régimen de seguridad respaldado por el grupo, ni presentar elementos que los aproximen o alejen en ese ámbito. Tampoco ha buscado investigar el poder relativo de cada país miembro del grupo. Según la visión de Bull, pretende mostrar, sin comprobarlo, la forma en que el poder conjunto de los BRICs influiría en la idea de que los estados miembros, como grupo, son un agente esencial para la gobernanza internacional, al igual que una gran potencia.

Se identificó la percepción interna del grupo en relación con un tema de la high politics, investigando la forma de introducir asuntos de seguridad en la pauta de sus reuniones de cúpula, así como llevar a cabo reuniones específicas dedicadas a seguridad. Así pues, se descubrió el constante aumento de los asuntos de seguridad en los documentos oficiales, aumento identificado en modo numérico comprobando el aumento del término "seguridad" en la secuencia de las declaraciones de cúpula efectuadas por el grupo, el que subió de 2 (cúpula I, 2009) a 43 (cúpula VII, 2015). También, en los análisis cualitativos de cada una de las declaraciones analizadas, detalladas en la última sección del artículo. Se infiere que tal situación puede significar un intento

${ }^{75}$ Véase Hurrell, op. cit. 
-discursivo en principio- de ganar legitimidad internacional en lo relativo a la materia.

Es de señalar que el estudio no deja de lado las varias divergencias entre los grupos, pero comprueba la idea de que, si por un lado hay controversias marcadas entre los miembros, por el otro procuran ampliar de manera estratégica sus demandas en áreas de convergencia.

Si bien es evidente el aumento de los temas sobre seguridad en las reuniones de los BRICS, son necesarios más estudios y una evaluación detallada. Es fundamental observar el desarrollo de las próximas declaraciones de cúpula que arrojarán más evidencias y así poder comprobar si la idea sobre el grupo se robustece o sucede lo contrario. Se busca confirmar la presente hipótesis con base en otras fuentes, sin perder de vista la limitación natural, aquí ya señalada, que conlleva una investigación restringida a documentos oficiales.

Traducción de Graciela Salazar

\section{BibLIOGRAFÍA}

"Asian Perspectives on Humanitarian Intervention in the $21^{\text {st }}$ Century", Doshisha 2nd International Conference on Humanitaria Intervention, 2012, en http://gdoshisha.files.wordpress.com/2012/06/sridhar-patnaik-paper.pdf

Baena Soares, João Clemente, "As Naçoes Unidas diante das Ameaças, dos desafios, das mudanças”, CEBRI Dossiê, vol. 1, año 4, 2005.

Baldwin, David, "The Concept of Security", Review of International Studies, núm. 23, 1997, pp. 5-26.

BRICS, I Declaración de cúpula del BRIC, 2009, en http//brics.itamaraty. gov.br/pt_br/categoría-portugues/20-documentos/73-primeiro-declaracao

— II Declaración de cúpula del BRIc, 2010, en http//brics.itamaraty.gov.br/pt_br/categoría-portugues/20-documentos/74-segundadeclaracao-conjunta 
, III Declaración de cúpula del BRICs, 2011, en http/ / www.itamaraty.gov.br/pt-BR/notas-a-imprensa/2559-iii-cupula-do-brics-sanya14-de-abril-de-2011

, IV Declaración de cúpula del BRICs, 2012, en http://brics.itamaraty.gov.br/pt_br/categoria-portugues/20-documentos/76-quarta-declaracao-conjunta

—, V Declaración de cúpula del BRIcs, 2013, en http://brics.itamaraty.gov.br/pt_br/sobre-o-brics/desenvolvimentos-recentes-v-cupula-durban-africa-do-sul-27-de-marco-de-2013

, VI Declaración de cúpula del BRICs, 2014, en http://brics.itamaraty.gov.br/pt_br/categoria-portugues/20-documentos/224-vicupula-declaracao-e-plano-de-acao-de-fortaleza

—, VII Declaración de cúpula del BRICs, 2015, en http//www.itamaraty.gov.br/pt-BR/notas-a-imprensa/10465-vii-cupula-do-brics-declaracao-de-ufa-ufa-russia-9-de-julho-de-2015

Bull, Hedley, A Sociedade Anárquica: um estudo da ordem na política mundial, trad. de Sérgio Bath, Brasilia, Editora Universidade de Brasilia, 2002.

Buzan, Barry, People, states and fear: An Agenda for security Analysis in the PostCold War Era, Brighton, Weatsheaf, 1991.

— , "An Underexploited Resource in IR", Review of International Studies, vol. 27, núm. 3, 2001, pp. 471-488.

$-\mathrm{y}$ Ole Waever, Regions and Powers. The Structure of International Security, Cambridge, University Press, 2003.

Cooper, Andrew F. y Asif B. Farooq, "BRICs and the Privileging of Informally in Global Governance”, Global Policy, vol. 4, núm. 4, 2013, pp. 428-432.

Fonseca, Pedro Cesar Dutra, Lucas de Oliveira Paes y André Moreira Cunha, "The concept of emerging power in international politics and economy”, Revista de Economia Política, vol. 36, núm. 1, 2016, pp. 46-69.

Fonseca Jr., Gelson, “BRIcs: notas e Questões”, en José Vicente de Sá Pimentel (org.), O Brasil, os BRICS e a Agenda Internacional, Brasilia, Funag, 2012.

Garwood-Gowers, Andrew, “China's «Responsible Protection» Concept: Reinterpreting the Responsibility to Protect (R2P) and Military Intervention for Humanitarian Purposes", Asian Journal of International Law, vol. 6, núm. 1, 2016, pp. 89-116. 
Gjorv, Gunhild Hoogensen, "Security by any other name: negative security, positive security, and a multi-actor security approach", Review of International Studies, vol. 38, núm. 4, 2012, pp. 836-839.

Herz, Monica y Victor Coutinho Lage, "Brics e a Questão Nuclear: contestações e reafirmações diante dos mecanismos de governança global", Carta Internacional, vol. 6, núm. 2, 2011, pp. 31-54.

Hour, Zhenbo, "The BRICS and Global Governance Reform: Can the BRICS provide leadership?”, Development, vol. 3, núm. 56, 2013, pp. 356-362.

Hurrell, Andrew, On Global Order: Power, Values, and the Constitution of International Society, Nueva York, Oxford University Press, 2007.

, "Hegemonia, liberalismo e ordem global: qual é o espaço para potências emergentes?”, en Andrew Hurrell, Maria R. Soares de Lima, Monica Hirst, Neil MacFarlane, Amrita Narlikar y Rosemary Foot, Os BRICS e a ordem global, Rio de Janeiro, Editora FGV, 2009.

, "Power Transitions, Emerging Powers, and the Shifting Terrain of the Middle Ground", en Cornelia Navari (ed.), Ethical Reasoning in International Affairs: Arguments from the Middle Ground, Londres, Palgrave Macmillan, 2013, pp. 222-245.

— Third World?", Brazilian Journal of Political Economy, vol. 33, núm. 2, 2013, pp. 203-221.

IPEA: Instituto de Pensamiento Europeo Ágora, O Fórum-2014, en http:// www.ipea.gov.br/forumbrics/pt-BR/o-forum.html

Laidi, Zaki, “BRICs: Sovereignty power and weaknes", International Politics, vol. 49, núm. 5, 2012, pp. 614-632.

Linklater, Andrew y Hidemi Suganami, The English School of International Relations: A Contemporary Reassessment, Cambridge, University Press, 2006.

O’Neill, Jim, "Building Better Global Economic BRIcs", GS Global Economics, Paper 66, noviembre de 2001, en http:/ /www.goldmansachs. com/our-thinking/archive/archive-pdfs/build-better-brics.pdf

Pérez, Lucas y Marianna Brito, "Os BRIcs na Votação do Projeto Europeu de Resolução do Conselho de Segurança Sobre a Síria”, Brics Policy Center - BRICs Monitor, noviembre de 2011, en http:/ / bricspolicycenter.org/homolog/publicacoes/Interna/881?tipo=Brics\%20Monitor

Reis, Maria Edileuza Fontenele, "BRICS: surgimento e evolução", en José Vicente de Sá Pimentel (org.), O Brasil, os BRICS e a Agenda Internacional, Brasilia, Funag, 2012, pp. 31-47. 
Stuenkel, Oliver, "The BRICS on the un Security Council: The Case of R2P", The Brics and the Future of Global Order, Lanhuam, Lexington Books, 2015, pp. 125-145.

Thakur, Ramesh, "R2P after Libya and Syria: Engaging Emerging Powers", The Washington Quarterly, vol. 36, núm. 2, 2013, pp. 61-76.

Troitskiy, Mikhail, "BRICS Approaches to Security Multilateralism", ASPJ Africa $\mathcal{E}$ Francophonie, $2^{\text {nd }}$ Quarter, 2015, pp. 76-88.

USCIRF: United States Commision on International Religious Freedom, "Annual Report of the United States Commission on International Religious Freedom”, en http://www.uscirf.gov/sites/default/files/ resources/annual1\%20report\%202010.pdf

Walt, Stephen M., "The end of the American era", The National Interest, núm. 116, 2011, pp. 6-16.

Zakaria, Fareed, O mundo pós-americano, trad. de Pedro Maia, São Paulo, Companhia das Letras, 2008. 